\title{
Spin Polarization of the Split Kondo State
}

\author{
Kirsten von Bergmann, ${ }^{1}$ Markus Ternes, ${ }^{2, *}$ Sebastian Loth, ${ }^{3,2}$ Christopher P. Lutz, ${ }^{4}$ and Andreas J. Heinrich ${ }^{4}$ \\ ${ }^{1}$ Department of Physics, University of Hamburg, 20355 Hamburg, Germany \\ ${ }^{2}$ Max-Planck Institute for Solid State Research, 70569 Stuttgart, Germany \\ ${ }^{3}$ Max-Planck Institute for the Structure and Dynamics of Matter, 22761 Hamburg, Germany \\ ${ }^{4}$ IBM Research Division, Almaden Research Center, San Jose, California 95120, USA
}

(Received 30 October 2014; published 20 February 2015)

\begin{abstract}
Spin-resolved scanning tunneling microscopy is employed to quantitatively determine the spin polarization of the magnetic field-split Kondo state. Tunneling conductance spectra of a Kondo-screened magnetic atom are evaluated within a simple model taking into account inelastic tunneling due to spin excitations and two Kondo peaks positioned symmetrically around the Fermi energy. We fit the spin state of the Kondo-screened atom with a spin Hamiltonian independent of the Kondo effect and account for Zeeman splitting of the Kondo peak in the magnetic field. We find that the width and the height of the Kondo peaks scales with the Zeeman energy. Our observations are consistent with full spin polarization of the Kondo peaks, i.e., a majority spin peak below the Fermi energy and a minority spin peak above.
\end{abstract}

PACS numbers: 72.15.Qm, 68.37.Ef

The Kondo effect was discovered experimentally nearly one century ago and starting from the 1960s theory has been employed to unravel its origin and properties [1]. It arises from the screening of a localized magnetic moment by host electrons, which leads to a Fermi-level resonance in the density of states. Kondo physics in the absence of a magnetic field has been studied extensively [2,3], whereas the precise behavior of the Kondo state in a magnetic field is less studied [4-8]. The coupling of the localized spin to the environment sets the relevant energy scale which is typically referred to as Kondo temperature $T_{K}$. For magnetic fields exceeding this energy the Kondo resonance splits. However, the spinresolved properties of this split Kondo state and, in particular, the amount of spin polarization of the two resulting peaks remains elusive $[9,10]$. While there is one spin-resolved measurement of a split Kondo state [11], the asymmetry of the peaks was not studied systematically and a comprehensive picture is missing.

The Kondo effect of a single atom or molecule on a surface can be probed with scanning tunneling spectroscopy (STS) [3]. When the localized spin is only weakly coupled to the conduction electrons of the substrate a perturbative description can be used [12] and a logarithmic peak at the Fermi energy is detected [13]. For stronger hybridization the arising correlations lead to a change in the density of states [6], which is typically described by a Lorentzian or a Frota function [14]. An additional linear tunnel channel gives rise to interference between different paths, leading to an asymmetric, Fano-like, line shape $[15,16]$. Systems with a spin $S>1 / 2$ can show the Kondo effect when the magnetocrystalline anisotropy leads to a degenerate $m= \pm 1 / 2$ ground state [5]. Then the resonance at zero bias is accompanied by steps in the tunnel spectra due to inelastic electron tunneling that excites the spin at finite energy. Inelastic tunnel spectroscopy has been employed to investigate such spin excitations in single atoms, molecules, and small clusters on ultrathin insulating layers, semiconductors, and metals [5,17-21].

In this Letter we quantitatively determine the spin polarization of the magnetic field-split Kondo state with spin-resolved STS in an external magnetic field. As a Kondo system we use a Co $S=3 / 2$ atom adsorbed on the $\mathrm{Cu}$ binding site of a thin $\mathrm{Cu}_{2} \mathrm{~N}$ layer, decoupling it from a metallic $\mathrm{Cu}(001)$ surface. The arising crystal field splitting leads to a hard anisotropy axis in the surface plane, perpendicular to the bonds to neighboring $\mathrm{N}$ atoms, and to a $m= \pm 1 / 2$ ground state of the adatom [5].

Figure 1(a) sketches the experimental setup of our spinpolarized STS measurements [22,23]. A magnetic field $B$ applied in the surface plane gives rise to two inequivalent Co atoms on the surface: magnetic field parallel to the hard anisotropy axis (denoted $\mathrm{CoV}$ ) and perpendicular to it (denoted $\mathrm{CoN}$ ). This enables measurements in inequivalent magnetic field directions with the same spin-polarized tip. Spectra were acquired at a temperature $T=0.5 \mathrm{~K}$ by recording the differential conductance as a function of sample bias, $d I / d V(V)$. The spectra in the absence of a magnetic field exhibit two distinct features [Fig. 1(a), inset]: a Kondo resonance at $E_{F}$ and spin-flip excitations appearing as steps at about $\pm 5 \mathrm{mV}$. In magnetic field the Kondo resonance splits and a double-peak emerges [Fig. 1(b) and 1(c)]. In addition, the energy of the spin excitation shifts and another low energy spin excitation due to a transition between $m= \pm 1 / 2$ states becomes possible, which is superimposed on the Kondo peaks. The spectra between the $\mathrm{CoV}$ and $\mathrm{CoN}$ differ in the amount of splitting and when measured with a non-spin-polarized (spin-averaging) tip, the spectra are almost mirror 

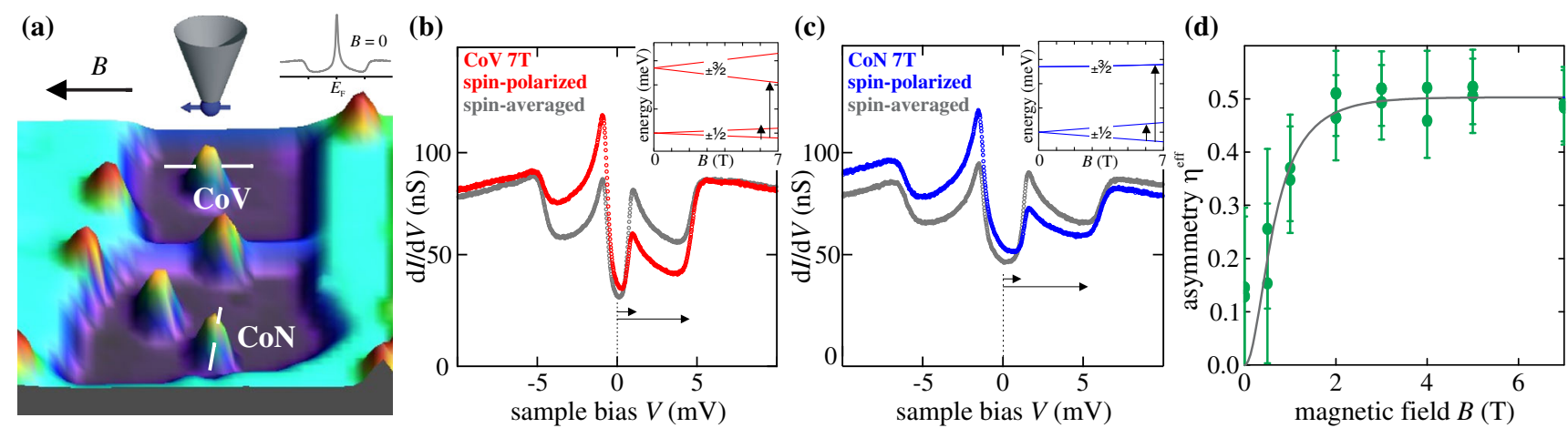

FIG. 1 (color online). (a) Setup of the spin-resolved STM experiment on individual Co atoms on $\mathrm{Cu}_{2} \mathrm{~N} / \mathrm{Cu}(001)$ in an in-plane magnetic field, STM topography image $(10 \mathrm{~nm} \times 10 \mathrm{~nm}, V=+10 \mathrm{mV}, I=0.1 \mathrm{nA})$; white lines denote the different uniaxial hard anisotropy axes perpendicular to each other; the right inset shows a $d I / d V$ spectrum of a Co atom at $B=0$. (b), (c) Spin-averaged (gray) and spin-resolved (color) tunnel spectra of $\mathrm{Co}$ atoms with magnetic field of $7 \mathrm{~T}$ applied along $(\mathrm{CoV})$ and perpendicular (CoN) to the hard anisotropy axis, respectively (stabilization set point $\sigma=80 \mathrm{nS}, V=+25 \mathrm{mV}, I=2 \mathrm{nA}$ ). Insets sketch the two relevant spin-flip excitations as a function of magnetic field. (d) The tip's spin polarization, deduced from measurements of a $\mathrm{Mn}$ atom on $\mathrm{Cu}_{2} \mathrm{~N}$, as a function of applied magnetic field; the solid line is a fit accounting for the paramagnetic behavior of the Mn atoms on tip and sample by Brillouin functions for spins with $S=5 / 2, g=2, T=0.5 \mathrm{~K}, \eta_{\text {sample }}=1, \eta_{\text {tip }}^{\max }=0.5$.

symmetric with respect to $0 \mathrm{~V}$ sample bias [24], whereas a spin-polarized tip leads to different step and peak heights for positive and negative bias. This asymmetry is due to the spin polarization of the tip in conjunction with the selections rules of the spin-flip transition [23]. The role of the Kondo effect for the asymmetry of the spectra is unclear and at the heart of this investigation.

The spin-polarized tip was prepared by picking up $\mathrm{Mn}$ atoms from the surface with the tip [23]. Since the measured asymmetry in the spectrum is the product of sample and tip spin polarization, $\eta^{\text {eff }}=\eta_{\text {sample }} \eta_{\text {tip }}$, it is crucial to characterize the degree of spin polarization of the tip. This was done by spin-resolved STS of individual Mn atoms on the same surface. $\mathrm{Mn}$ atoms on $\mathrm{Cu}_{2} \mathrm{~N}$ show one spin-flip excitation at about $1 \mathrm{mV}$ [18]. When measured with a spin-polarized tip, the heights of the inelastic steps at positive and negative voltage $\left(h^{+}\right.$and $\left.h^{-}\right)$differ [23]. The asymmetry of the step heights $\eta^{\text {eff }}=\left(h^{-}-h^{+}\right) /\left(h^{-}+h^{+}\right)$ for two individual $\mathrm{Mn}$ atoms is measured as a function of magnetic field. Because of the small magnetocrystalline anisotropy of $\mathrm{Mn}$ the nominal spin polarization of the step is $\eta_{\mathrm{Mn}}=1$, and therefore in this case the experimental $\eta^{\text {eff }}$ is a quantitative measure of the tip spin polarization $\eta_{\text {tip }}$. We find that the magnetic-field dependence of the tip's spin polarization is consistent with paramagnetic behavior of the Mn atoms on the sample and the metallic tip. Hence magnetic field-dependent spin polarization of the tip and sample are well described by Brillouin functions [solid line in Fig. 1(d)]. In the following we use this functional dependence to account for the field dependence of the tip's spin polarization.

To determine the spin polarization of the split Kondo state quantitatively it is necessary to disentangle the spinflip excitation features and the Kondo resonance in the tunnel spectra. We fit the experimental data with a simple model: we consider for each tunnel spectrum two spin-flip transitions and model the Kondo peaks by Frota functions [Figs. 2(a) and 2(b)]. The full spectrum is therefore

$$
\begin{aligned}
\sigma(e V)= & \sigma_{0}+h_{i}^{-} \theta\left(\varepsilon_{i}+e V\right)+h_{i}^{+} \theta\left(\varepsilon_{i}-e V\right) \\
& +h_{o}^{-} \theta\left(\varepsilon_{o}+e V\right)+h_{o}^{+} \theta\left(\varepsilon_{o}-e V\right) \\
& +h_{K}^{-} g\left(\varepsilon_{K}+e V, \Gamma_{K}\right)+h_{K}^{+} g\left(\varepsilon_{K}-e V, \Gamma_{K}\right),
\end{aligned}
$$

where $\theta$ is the temperature-broadened step function

$$
\theta(\varepsilon)=\frac{1+(x-1) \exp (x)}{(\exp (x)-1)^{2}}
$$

with $x=\left(\varepsilon / k_{B} T\right)$, and $g$ is the Frota function $[7,13,16]$

$$
g\left(\varepsilon, \Gamma_{F}\right)=\Re\left(\sqrt{\frac{i \Gamma_{F}}{i \Gamma_{F}+\varepsilon}}\right),
$$

with $\Re(x)$ as the real part of $x$ and where the half-width at half-maximum of the peak is given by $\Gamma_{K}=2.54 \Gamma_{F}$ [14]. At temperatures $T \ll T_{K}$ and $B=0$ it is directly related to the Kondo temperature $T_{K}=\Gamma_{K}^{0} / k_{B}$ via the Boltzmann constant $k_{B}$. The "outer" step at around $\varepsilon_{o}= \pm 5 \mathrm{mV}$ with height $h_{o}$ corresponds to the excitation from $\pm 1 / 2$ to $\pm 3 / 2$ states; the "inner" step with $h_{i}$ occurs between the $\pm 1 / 2$ states and is found at an energy $\varepsilon_{i}<1 \mathrm{meV}$. Based on previous work [5] we introduce the constraint that the Kondo peak energy is identical to the energy of the $\pm 1 / 2$ transition, $\varepsilon_{K}=\varepsilon_{i}$, and perform a least-squares fit of all remaining parameters allowing for a vertical offset $\sigma_{0}$.

The step energies $\varepsilon_{o}$ and $\varepsilon_{i}$ resulting from the fits serve now as input parameters for a calculation with a model spin Hamiltonian for each Co atom accounting for Zeeman splitting, uniaxial, and transverse magnetic anisotropy, 

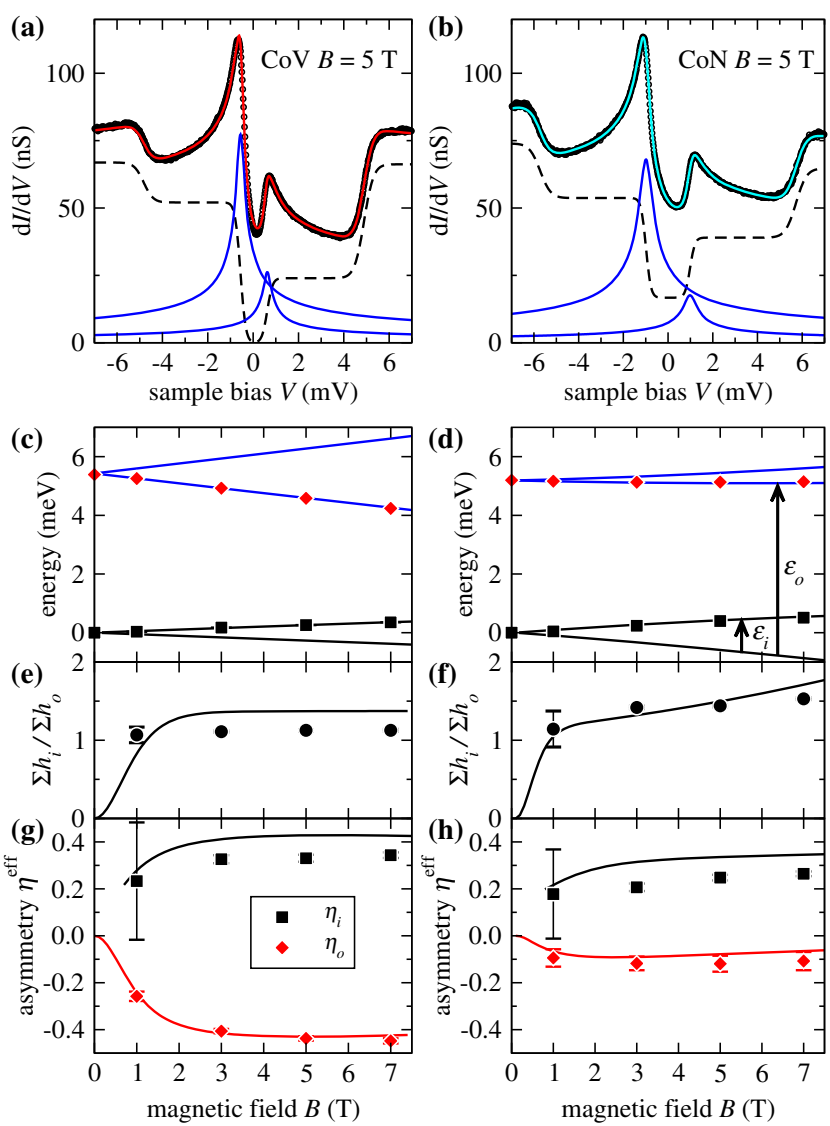

FIG. 2 (color online). Decomposition of the $d I / d V$ spectra into spin-flip excitation and Kondo related contributions. (a),(b) Experimental data (circles) measured on a $\mathrm{CoV}$ and a $\mathrm{CoN}$ atom at $B=5 \mathrm{~T}$ (stabilization set point $\sigma=80 \mathrm{nS}, V=+25 \mathrm{mV}$, $I=2 \mathrm{nA}$ ). Upper solid line: best fit using the sum of an asymmetric double step function (dashed line) and two Frota functions (lower solid lines), according to Eq. (1) of the main text. (c),(d) Energies $\varepsilon_{i, o}$, (e),(f) ratio of the intensities $\left(h_{i}^{+}+h_{i}^{-}\right) /\left(h_{o}^{+}+h_{o}^{-}\right)$, and $(\mathrm{g}),(\mathrm{h})$ effective asymmetries of the two steps at different magnetic field. Symbols are fits to the data [25]. Lines are calculated with the model spin Hamiltonian using $\quad D=2.6 \pm 0.1 \quad(2.5 \pm 0.1) \mathrm{meV}, \quad E=0.45 \pm 0.05$ $(0.40 \pm 0.05) \mathrm{meV}$, and $g=2.1 \pm 0.1(2.4 \pm 0.1)$ for the $\mathrm{CoV}$ $(\mathrm{CoN})$ atom.

$$
\hat{H}=g \mu_{B} \vec{B} \hat{\mathbf{S}}+D \hat{S}_{z}^{2}+E\left(\hat{S}_{x}^{2}+\hat{S}_{y}^{2}\right) .
$$

The curves in Figs. 2(c) and 2(d) show the magnetic field dependence of $\varepsilon_{o}$ and $\varepsilon_{i}$ for the derived values of the magnetic anisotropy parameters $D, E$ and the $g$ factor (see caption Fig. 2). The curves in Figs. 2(e), 2(f) and 2(g), 2(h) are the calculated ratio between inner and outer step intensities and the effective step asymmetries for the $\mathrm{CoV}$ and $\mathrm{CoN}$ atoms, respectively. In our model $\eta^{\text {eff }}$ is the product of the tip polarization and the polarization of the spin excitation calculated from the transition matrix elements for the spin Hamiltonian [26]. Whereas the measured step asymmetry $\eta_{o}$ extracted from the spectra agrees well with the calculated effective asymmetry, the experimental values of $\eta_{i}$ are slightly smaller than the calculated ones. This is possibly caused by a competition between spin-flip excitation and Kondo scattering not accounted for in the model. Nevertheless, considering the overall agreement between the fits to the tunnel spectra and the calculation, we conclude that we can capture well the contributions from inelastic spin-flip excitations with this simple model spin Hamiltonian, similar to previous work [5,17-21].

We now turn to our initial question about the spin polarization of the split Kondo peak. Figures 3(a) and 3(b) present the Kondo-related spectra, i.e., the difference between the original data and the spin-flip excitation contributions. It becomes apparent that the asymmetry at $\pm \varepsilon_{K}$ is not only due to the polarization of the spin-flip excitation step, but that indeed there is a significant asymmetry of the Kondo peaks. We find that the peak
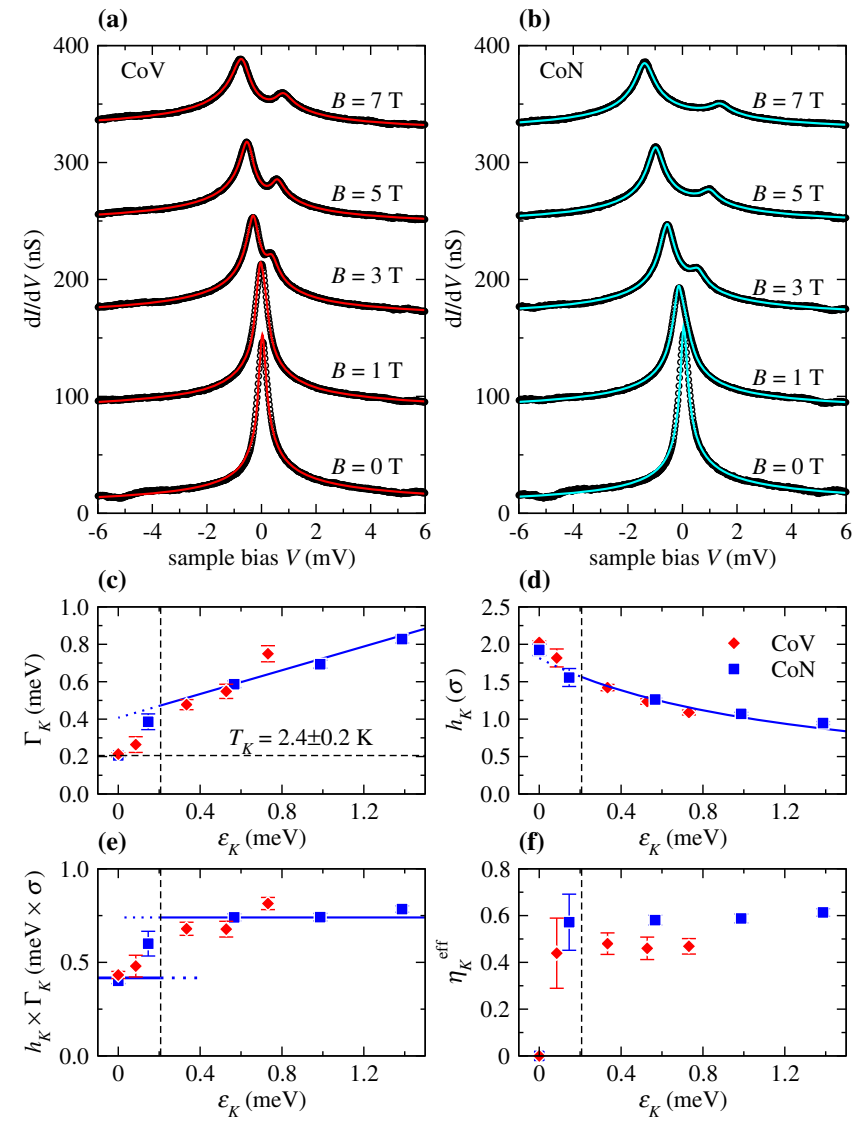

FIG. 3 (color online). The splitting and the polarization of the Kondo peak. (a),(b) Kondo-related experimental data (circles) and fit with two Frota functions (solid lines) for $\mathrm{CoV}$ and $\mathrm{CoN}$ (curves in magnetic field are shifted vertically for better visualization). Fit results for (c) the width $\Gamma_{K}$, (d) the peak height $h_{K}$, (e) the area $\Gamma_{K} h_{K}$, and (f) the experimental peak asymmetry $\eta_{K}^{\text {eff }}$ plotted against the splitting energy $\varepsilon_{K}$ [25]. The horizontal and vertical dashed lines in (c)-(f) mark the characteristic Kondo energy. 
width $\Gamma_{K}$ increases, Fig. 3(c), and the peak height $h_{K}$ drops, Fig. 3(d), with increasing magnetic field for both $\mathrm{CoV}$ and $\mathrm{CoN}$, as expected from numerical renormalization group theory calculations [6-8]. Interestingly, the behavior of the Co atoms seems to be related to the splitting energy as the data for $\mathrm{CoV}$ and $\mathrm{CoN}$ fall on top of each other when plotted versus the peak energy $\varepsilon_{K}$ leading to a $1 / \varepsilon_{K}$ dependence of the peak height $h_{K}$. From the peak width at $B=0\left(\Gamma_{K}^{0}\right)$ the Kondo temperature is extracted to $T_{K}=2.4 \pm 0.2 \mathrm{~K}$, which is equivalent to an energy of $k_{B} T_{K}=0.21 \mathrm{meV}$. When the splitting exceeds this energy, we observe that the spectral weight, i.e., the area $h_{k} \times \Gamma_{K}$ under the Kondo peak, settles at about twice the zero field value, and remains afterward constant irrespective of magnetic field up to $7 \mathrm{~T}$ [Fig. 3(e)]. Presumably, this stems from the lifting of the spin degeneracy of the Kondo singlet state at significant field.

In this regime, where $\varepsilon_{K}>k_{B} T_{K}$, our data suggest a linear dependence of $\Gamma_{K}$ on $\varepsilon_{K}$, which leads to a surprisingly simple equation:

$$
\Gamma_{K}\left(\varepsilon_{K}\right)=(2 \pm 0.1) \Gamma_{K}^{0}+(1 \pm 0.03) \frac{1}{\pi} \varepsilon_{K},
$$

where we associate the linear term to an increased scattering with bulk electrons which reduces the lifetime [23]. Figure 3(f) demonstrates that the effective polarization of the Kondo peak $\eta_{\mathrm{K}}^{\text {eff }}$ for both $\mathrm{CoV}$ and $\mathrm{CoN}$ stays constant and is close to 0.5 , with a small systematic offset between the values for $\mathrm{CoV}$ and $\mathrm{CoN}$.

Finally, in order to derive the spin polarization of the split Kondo peak $\eta_{\mathrm{K}}$, we need to consider the spin-polarization of the tip $\left(\eta_{\mathrm{K}}=\eta_{\mathrm{K}}^{\text {eff }} / \eta_{\text {tip }}\right)$. Figure 4(a) shows the resulting spin-polarization of the split Kondo peak $\eta_{\mathrm{K}}$ and we find that it scatters around one. This key finding indicates that the Kondo feature is fully spin polarized, meaning that the peak at negative sample bias, i.e., occupied density of states, is entirely in the majority spin state and the peak at positive bias, i.e., unoccupied states, is entirely in the minority spin state. The implication for a tunnel spectroscopy experiment in an external magnetic field is illustrated in Fig. 4(b): when the tip polarization is 0, two Kondo peaks with equal height are measured, whereas a fully spinpolarized tip with $\eta_{\text {tip }}=1$ would lead to a single peak at $-\varepsilon_{K}$. We note that the average spin-polarization $\eta_{K}$ for $\mathrm{CoN}$ exceeds the value of one with statistical significance. This might point out the limits of our analysis. It suggests a peak-dip structure in the spectrum, see the dashed line in Fig. 4(b), which might stem from a non-negligible ferromagnetic Kondo component in this high spin system [27].

To conclude, our experiments show that the magnetic field-split Kondo state can be described by two independent fully spin-polarized peaks. For magnetic fields that exceed the characteristic Kondo energy, the width of the peaks increases linearly with the splitting energy, and together with a decreasing height this leads to a constant spectral

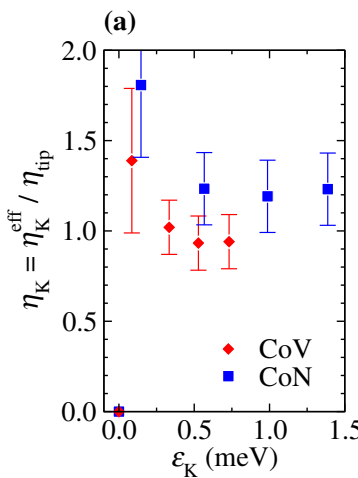

(b)

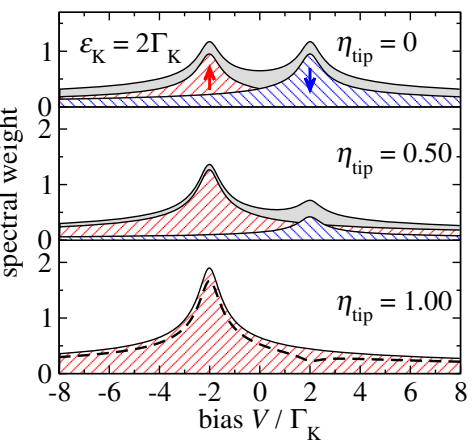

FIG. 4 (color online). (a) The spin polarization of the Kondo state $\eta_{K}$ is approximately one for both $\mathrm{CoV}$ and $\mathrm{CoN}$ independent of $\varepsilon_{K}$. (b) Simulated spectra with $\eta_{K}=1$ and a magnetic field which splits the Kondo state by $\varepsilon_{K}=2 \Gamma_{K}$ for different tip spin polarizations; gray is the sum of the majority and minority states, i.e., the experimentally measured spectrum. The dashed line in the lower panel shows the peak-dip structure expected for an apparent $\eta_{K}=1.2$.

weight. These experimental results can serve as a reference for further theory studies of the spin-resolved behavior of the Kondo effect in an external magnetic field. In addition, the field-split Kondo state could be exploited to serve as a magnetic probe in transport measurements, similar to the fully spin-polarized magnetic field split superconducting state $[28,29]$. Experimentally, this could be realized, for example, by attaching a magnetic molecule that exhibits a Kondo resonance to the tip apex. The Kondo resonance would then act as an energy-dependent spin filter for quantitative spin-resolved STM measurements.

K. v. B. acknowledges financial support from the German research foundation (DFG Forschungsstipendium), S.L. from the Alexander von Humboldt foundation, A.J.H. and C.P.L. from the Office of Naval Research.

*m.ternes@fkf.mpg.de

[1] A. C. Hewson, The Kondo Problem to Heavy Fermions (Cambridge University Press, Cambridge, England, 1997).

[2] M. Pustilnik and L. Glazman, J. Phys. Condens. Matter 16, R513 (2004).

[3] M. Ternes, A. J. Heinrich, and W.-D. Schneider, J. Phys. Condens. Matter 21, 053001 (2009).

[4] C. H. L. Quay, J. Cumings, S. J. Gamble, R. dePicciotto, H. Kataura, and D. Goldhaber-Gordon, Phys. Rev. B 76, 245311 (2007).

[5] A. F. Otte, M. Ternes, K. von Bergmann, S. Loth, H. Brune, C. P. Lutz, C. F. Hirjibehedin, and A. J. Heinrich, Nat. Phys. 4, 847 (2008).

[6] T. A. Costi, Phys. Rev. Lett. 85, 1504 (2000).

[7] R. Zitko, R. Peters, and T. Pruschke, New J. Phys. 11, 053003 (2009). 
[8] S. Smirnov and M. Grifoni, New J. Phys. 15, 073047 (2013).

[9] K. R. Patton, S. Kettemann, A. Zhuravlev, and A. Lichtenstein, Phys. Rev. B 76, 100408 (2007).

[10] A. C. Seridonio, F. M. Souza, and I. A. Shelykh, J. Phys. Condens. Matter 21, 095003 (2009).

[11] Y.-S. Fu, Q.-K. Xue, and R. Wiesendanger, Phys. Rev. Lett. 108, 087203 (2012).

[12] J. A. Appelbaum, Phys. Rev. 154, 633 (1967).

[13] Y.-h. Zhang, S. Kahle, T. Herden, C. Stroh, M. Mayor, U. Schlickum, M. Ternes, P. Wahl, and K. Kern, Nat. Commun. 4, 2110 (2013).

[14] H. O. Frota, Phys. Rev. B 45, 1096 (1992).

[15] V. Madhavan, W. Chen, T. Jamneala, M. F. Crommie, and N. S. Wingreen, Science 280, 567 (1998).

[16] H. Prüser, M. Wenderoth, P. E. Dargel, A. Weismann, R. Peters, T. Pruschke, and R. G. Ulbrich, Nat. Phys. 7, 203 (2011).

[17] A. J. Heinrich, J. Gupta, C. P. Lutz, and D. M. Eigler, Science 306, 466 (2004).

[18] C. F. Hirjibehedin, C. P. Lutz, and A. J. Heinrich, Science 312, 1021 (2006).

[19] A. A. Khajetoorians, B. Chilian, J. Wiebe, S. Schuwalow, F. Lechermann, and R. Wiesendanger, Nature (London) 467, 1084 (2010).
[20] A. A. Khajetoorians, S. Lounis, B. Chilian, A. T. Costa, L. Zhou, D. L. Mills, J. Wiebe, and R. Wiesendanger, Phys. Rev. Lett. 106, 037205 (2011).

[21] S. Kahle, Z. Deng, N. Malinowski, C. Tonnoir, A. FormentAliaga, N. Thontasen, G. Rinke, D. Le, V. Turkowski, T. S. Rahman, S. Rauschenbach, M. Ternes, and K. Kern, Nano Lett. 12, 518 (2012).

[22] R. Wiesendanger, Rev. Mod. Phys. 81, 1495 (2009).

[23] S. Loth, K. von Bergmann, M. Ternes, A. F. Otte, C. P. Lutz, and A. J. Heinrich, Nat. Phys. 6, 340 (2010).

[24] The asymmetry of the peak heights in the spin-averaged measurement is $<4 \%$ and might be due to some residual spin polarization in the tip or resonant elastic scattering.

[25] Error bars indicate the $90 \%$ confidence interval of the fit and stem mainly from the interdependence of the parameters (nonzero off-diagonal covariance matrix elements).

[26] S. Loth, C. P. Lutz, and A. J. Heinrich, New J. Phys. 12, 125021 (2010).

[27] P. P. Baruselli, R. Requist, M. Fabrizio, and E. Tosatti, Phys. Rev. Lett. 111, 047201 (2013).

[28] R. Meservey and P. Tedrow, Phys. Rep. 238, 173 (1994).

[29] M. Eltschka, B. Jäck, M. Assig, O. V. Kondrashov, M. A. Skvortsov, M. Etzkorn, C. R. Ast, and K. Kern, Nano Lett. 14, 7171 (2014). 